\title{
Editorial
}

\section{Advanced Sensor Technologies in Geospatial Sciences and Engineering}

\author{
Hyung-Sup Jung $\mathbb{D},{ }^{1}$ Lei Zhang $\mathbb{D}^{2},{ }^{2}$ and Sang-Hoon Hong $\mathbb{D}^{3}$ \\ ${ }^{1}$ Department of Geoinformatics, The University of Seoul, Seoul, Republic of Korea \\ ${ }^{2}$ Department of Land Surveying and Geo-Informatics, The Hong Kong Polytechnic University, Hung Hom, Hong Kong \\ ${ }^{3}$ Department of Geological Sciences, Pusan National University, Busan, Republic of Korea
}

Correspondence should be addressed to Sang-Hoon Hong; geoshong@pusan.ac.kr

Received 19 August 2019; Accepted 22 August 2019; Published 4 September 2019

Copyright (c) 2019 Hyung-Sup Jung et al. This is an open access article distributed under the Creative Commons Attribution License, which permits unrestricted use, distribution, and reproduction in any medium, provided the original work is properly cited.

Advanced sensor technologies in geospatial sciences and engineering have become more essential to (1) contributing to geographic mapping and (2) understanding geological, ecological, hydrological, and environmental characteristics of Earth surfaces. Among 56 papers submitted to this special issue, 24 original research articles, which address the advanced sensor technologies in geospatial sciences and engineering, have been published. The published papers can be summarized as follows.

N.-W. Park and P. C. Kyriakidis (2019) in their paper entitled "A Geostatistical Approach to Spatial Quality Assessment of Coarse Spatial Resolution Remote Sensing Products" showed a geostatistical framework for spatial quality assessment of coarse resolution satellite-based products using point-level validation data and geostatistical simulation.

F. Hu et al. (2019) in their paper entitled "Dynamic Linear Predictive Optimization of Flexible Robot Profiling MFA Model" proposed a flexible robot profiling ModelFree Adaptive (MFA) model based on adaptive predictive dynamic linear optimization. The authors claimed that the flexible robot profiling MFA model could establish the realtime control of airbag charging and discharging deformation profiling of the flexible robot.

K.-Y. Oh et al. (2019) in their paper entitled "Quality Assessment of Four DEMs Generated Using In-Track KOMPSAT-3 Stereo Images" analyzed the quality and characteristics of four digital elevation models (DEMs) generated using in-orbit KOrea Multi-Purpose SATellite-3 (KOMPSAT-3) stereo images. Their result showed that the sufficient-qualified DEM could be generated from the KOMPSAT-3 stereo images without ground control points (GCPs).

J. Dudak et al. (2019) in their paper entitled "Implementation of Secure Communication via the RF Module for Data Acquisition" described the use of the VirtualWire communication protocol using radio frequency waves with a carrier frequency of $433 \mathrm{MHz}$ to $900 \mathrm{MHz}$. This paper focused on the design and implementation of a secure one-way communication channel.

B. Hu et al. (2019) in their paper entitled "Time-Series Displacement of Land Subsidence in Fuzhou Downtown, Monitored by SBAS-InSAR Technique" monitored the land subsidence in the Fuzhou downtown after the metro construction program using multitemporal synthetic aperture radar (SAR) interferometry (MT-InSAR). For the study, they used 24 scenes of X-band TerraSAR-X data from July 2013 to August 2015 and 32 scenes of C-band Sentinel-1 data from July 2015 to February 2018. Their results showed that (i) the maximum subsidence rate was about $-12 \mathrm{~mm} / \mathrm{yr}$ and (ii) eight subsidence funnels were found during the observed period.

J. Han et al. (2019) in their paper entitled "Preliminary Results of the Development of a Single-Frequency GNSS RTK-Based Autonomous Driving System for a Speed Sprayer" developed an autonomous driving system using a 
single-frequency GNSS RTK for commercialization of an autonomous driving speed sprayer. The field test of the developed system achieved accuracy of about $0.01 \mathrm{~m}$.

Y. Xie et al. (2019) in their paper entitled "A Novel Convolutional Neural Network Architecture for SAR Target Recognition" proposed a novel convolutional neural network (CNN) architecture suitable for the SAR data through improving the Inception-ResNet architecture from the optical images. Their result showed that the proposed architecture achieved (i) higher than 99\% accuracy for the classification of 10-class targets and (ii) higher than $96 \%$ accuracy for the classification of eight variants of the chosen target.

$\mathrm{H}$. Wu et al. (2019) in their paper entitled "Concrete Spalling Detection for Metro Tunnel from Point Cloud Based on Roughness Descriptor" proposed a novel method that can automatically detect the concrete spalling damage on the tunnel surface from 3D point clouds obtained by a mobile laser scanning system. The proposed method has two characteristics: (i) automatic concrete spalling detection for tunnel surfaces and (ii) guidelines for choosing optimal scanning parameters.

K.-H. Kim et al. (2019) in their paper entitled "Investigation of Coastal Environment Change Using Wave Measurement Sensors and Geographical Laser Scanner" analyzed the cause and mechanism of damage inflicted on coasts by artificial structures through reviewing beach erosions that have occurred on beaches on the east coast of Korea after the large-scale construction of artificial structures.

S. Zhang et al. (2019) in their paper entitled "UAV-Based Gigapixel Panoramic Image Acquisition Planning with Ray Casting-Based Overlap Constraints" proposed a gigapixel panorama acquisition planning method for multirotors. It is based on a ray casting image overlap calculation method.

D. Qiu et al. (2019) in their paper entitled "A Deformation Prediction Approach for Supertall Building Using Sensor Monitoring System" applied the integration of the levenberg-marquardt (LM) and the conditional deep belief network (CDBN) model to the deformation prediction of the CITIC tower, the tallest building in Beijing. They verified the prediction accuracy and stability of the proposed model through model comparison experiments and predictive analysis.

D.-C. Lee et al. (2019) in their paper entitled "Determination of Building Model Key Points Using Multidirectional Shaded Relief Images Generated from Airborne LiDAR Data" implemented the curvature scale space (CSS) algorithm to determine model key points (MKPs) of the buildings with various roof types.

S.-Y. Park et al. (2019) in their paper entitled "Segmentation of LiDAR Data Using Multilevel Cube Code" introduced a shape descriptor method for segmenting LiDAR point clouds using a "multilevel cube code" that is an extension of the $2 \mathrm{D}$ chain code to $3 \mathrm{D}$ space. The cube operator segments point clouds into roof surface patches, including superstructures, removes unnecessary objects, detects the boundaries of buildings, and determines model key points for building modeling. The proposed method was validated by using both real and simulated LiDAR data.
J. Shin et al. (2019) in their paper entitled "Characteristics of Laser Backscattering Intensity to Detect Frozen and Wet Surfaces on Roads" investigated the signal characteristics of laser backscattering intensity in order to detect frozen and wet surfaces on roads.

T.-S. Bae and C.-K. Hong (2019) in their paper entitled "Analysis of Parameter Correlations of the ECOM Solar Radiation Pressure Model for GPS Orbit" analyzed the global positioning system (GPS) orbit model in terms of the correlation between solar radiation pressure (SRP) parameters and the residuals of orbit estimation.

Y. Han et al. (2019) in their paper entitled "Automated Coregistration of Multisensor Orthophotos Generated from Unmanned Aerial Vehicle Platforms" proposed an automated coregistration approach for orthophotos generated from very-high-resolution (VHR) images acquired from multisensors mounted on unmanned aerial vehicle (UAV) platforms.

X. Zhu et al. (2019) in their paper entitled "Topological Characteristics and Vulnerability Analysis of Rural Traffic Network" built a proper rural rating system in China, including National Road, Provincial Road, County Road, Rural Road, and Special Road. The authors modeled three static networks of No-power Traffic Network Model (NTNM), Distance Weight Traffic Network Model (DWTNM), and Road Level Weight Traffic Network Model (RLWTNM) to explore the vulnerability of the rural traffic network (RTN) in Zhangwu in China.

B. Hu et al. (2019) in their paper entitled "Monitoring the Thaw Slump-Derived Thermokarst in the Qinghai-Tibet Plateau Using Satellite SAR Interferometry" investigated and validated the distributions and active level of the thawinduced slope failures with field observations, quantifying spatial-temporal variability in surface deformation at thaw slumps from 2007 to 2010. The authors utilized time series SAR interferometry (InSAR) application to map and study thaw slumps in the Qinghai-Tibet plateau (QTP), which have not been well investigated in previous studies.

J. Wang et al. (2019) in their paper entitled "Demonstration of Time-Series InSAR Processing in Beijing Using a Small Stack of Gaofen-3 Differential Interferograms" investigated the surface deformation of the metropolitan area in Beijing from March 2017 to January 2018 using five Gaofen-3 (GF-3) SAR. The study evaluates the interferometric capability of GF-3 SAR data.

H.-U. Kim and T.-S. Bae (2019) in their paper entitled "Deep Learning-Based GNSS Network-Based Real-Time Kinematic Improvement for Autonomous Ground Vehicle Navigation" developed a model to estimate the position of the vehicle without redesigning the analytical model of each individual sensor with constraints. The authors applied the deep learning technique to predict the position of the vehicle based on multisensor data including GNSS. The field experiment was carried out using the mobile mapping system (MMS).

M. E. H. Chowdhury et al. (2019) in their paper entitled "A Low-Cost Closed-Loop Solar Tracking System Based on the Sun Position Algorithm" developed a simple, costeffective algorithm-based reliable two-axis tracking system 
for real-time solar position measurement on an 8-bit microcontroller platform.

B. Hu et al. (2019) in their paper entitled "Using Multisensor SAR Datasets to Monitor Land Subsidence in Los Angeles from 2003 to 2017" mapped the temporal and spatial variations of land subsidence in Los Angeles. The authors used an improved small baseline subset (SBAS) technique and multisensor SAR datasets to analyze the causes of deformations from October 2003 to October 2017.

J. Liu et al. (2019) in their paper entitled "Neural Network with Confidence Kernel for Robust Vibration Frequency Prediction" proposed a novel noncontact machine learningbased system to predict directly vibration frequency with high accuracy and good reliability by using image sequences acquired from a single camera.

Q. Zhang et al. (2018) in their paper entitled "Precipitable Water Vapor Retrieval and Analysis by Multiple Data Sources: Ground-Based GNSS, Radio Occultation, Radiosonde, Microwave Satellite, and NWP Reanalysis Data" presented a case of retrieved precipitable water vapor (PWV) using microwave satellite observation.

\section{Conflicts of Interest}

The Guest Editors declare that there is no conflict of interest regarding the publication of this article.

\section{Acknowledgments}

We would like to thank all the authors for their valuable contributions in this special issue as well as all the reviewers who are all experts on the theme. And also, we would like to thank all members of the editorial board for approving this special issue. This work was supported by the National Research Foundation of Korea (NRF) grant funded by the Korea government (MSIT) (No. 2019R1F1A1059570), and it was also supported by Korea Agency for Infrastructure Technology Advancement (KAIA) grant funded by the Korea Government (MOLIT) (No. 19TLRP-C145770-02, Development of Drone-Based Technology for Efficient Maintenance of Cutting Slope and Pavement).

Hyung-Sup Jung Lei Zhang

Sang-Hoon Hong 


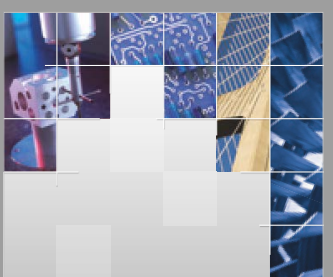

\section{Enfincering}
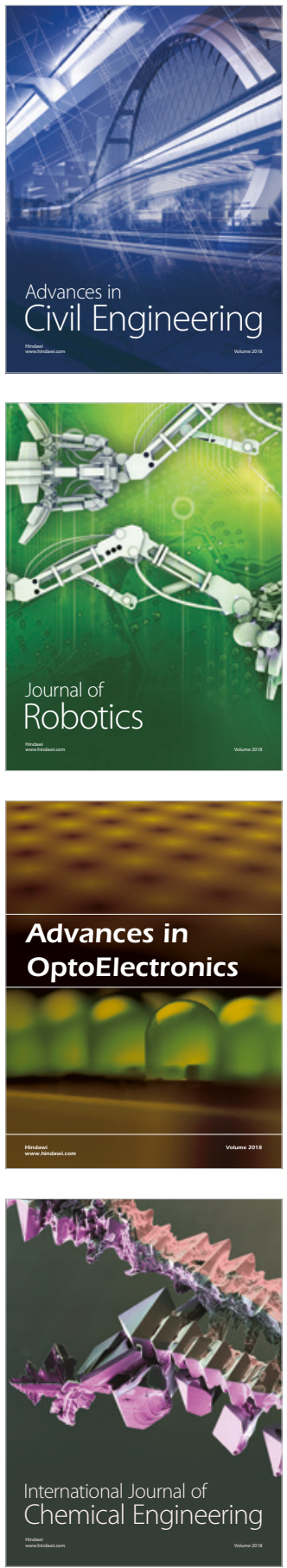

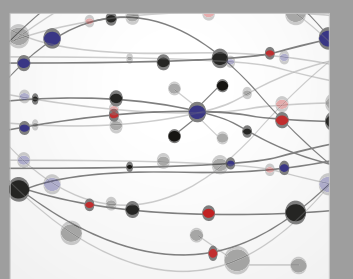

\section{Rotating \\ Machinery}

The Scientific World Journal

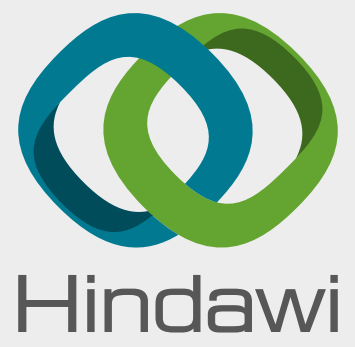

Submit your manuscripts at

www.hindawi.com
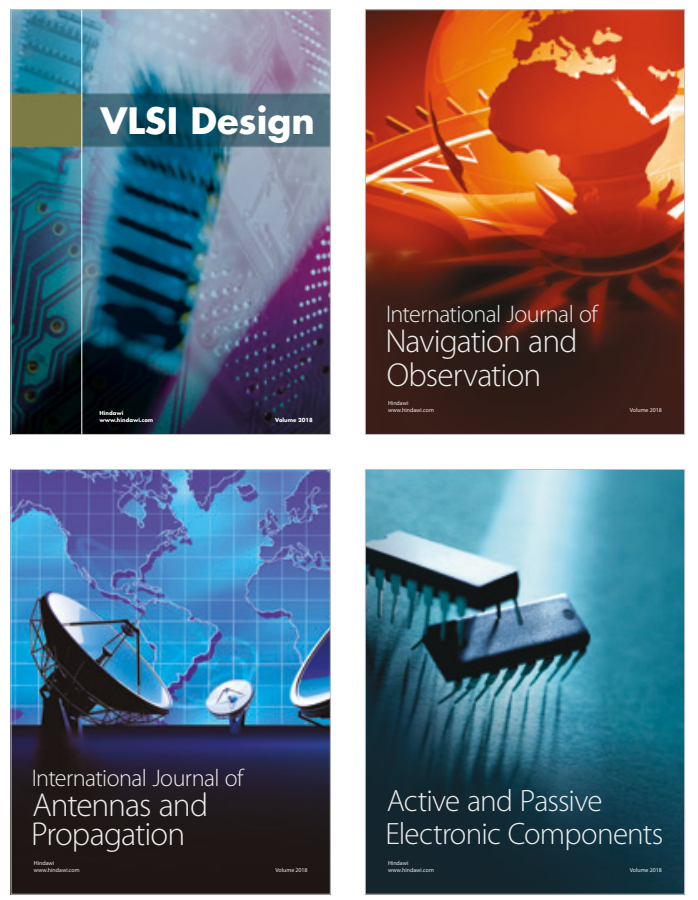
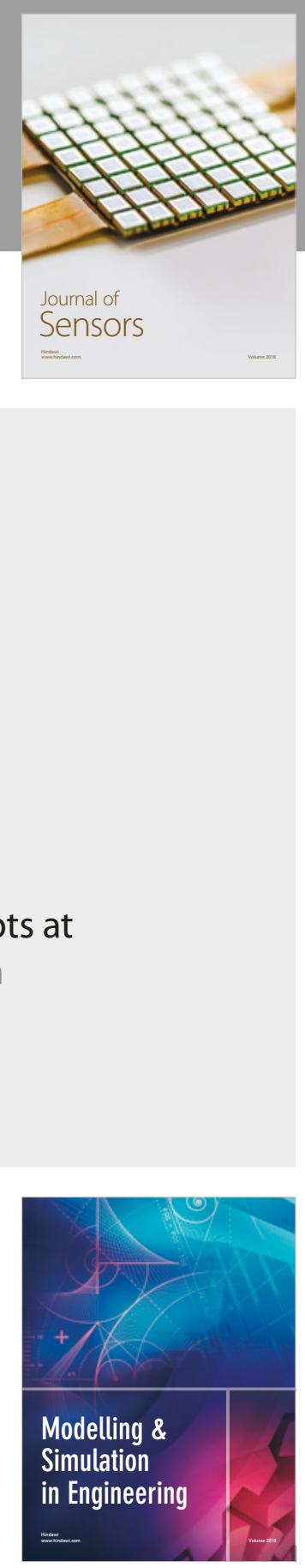

\section{Advances \\ Multimedia}
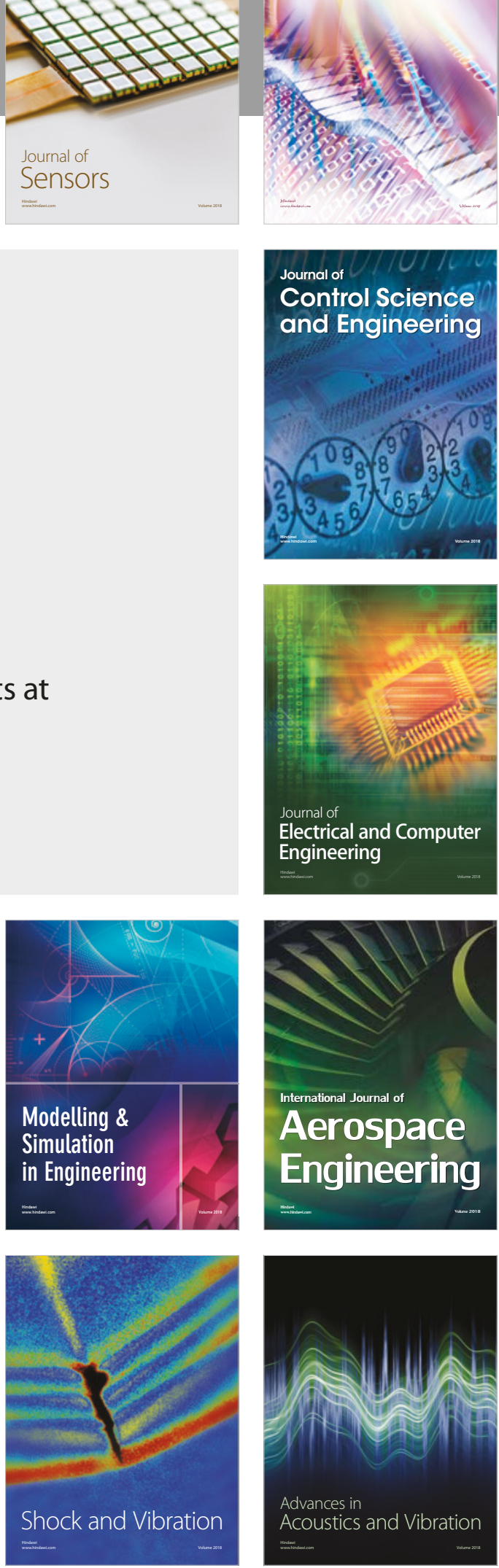